\title{
Disección atrial izquierda: Características ecocardiográficas y el aporte de la multimodalidad
}

\author{
Gabriela Guevara-Castilla \\ Luis Murillo-Pérez²
}

\section{Correspondencia}

Gabriela Guevara Castilla

gabrielaguevc@yahoo.com

${ }^{\top}$ Médico asistente del Servicio de Cardiología No Invasiva. Instituto Nacional Cardiovascular INCOR - EsSalud. Lima. Perú.

${ }^{2}$ Médico residente de cardiología. Instituto Nacional Cardiovascular INCOR - EsSalud. Lima. Perú.

Enviado: 10/10/2021.

Aceptado: 05/11/2021.

Publicado: 15/12/2021.

Citar como: Guevara Castilla G., Murillo Perez L. Disección atrial izquierda: Características ecocardiográficas y el aporte de la multimodalidad. RETIC. 2021(Diciembre); 4 (3): 28-31. doi: 10.37615/retic.v4n3a8

Cite this as: Guevara Castilla G., Murillo Perez L. Left atrial disection: Echocardiographic characteristics and multimodality input. RETIC. 2021 (December); 4 (3): 28-31. doi: 10.37615/retic.v4n3a8

\section{Palabras clave \\ $\triangleright$ Disección atrial. \\ $\triangleright$ Ecocardiografía transesofágica. \\ $\triangleright$ Tomografía cardiaca. \\ $\triangleright$ Multimodalidad.}

\begin{abstract}
RESUMEN
La disección atrial es una entidad rara que ocurre generalmente como complicación de una cirugía de la válvula mitral. La ecocardiografía transesofágica constituye la herramienta diagnóstica más importante, sin embargo, actualmente contamos con diferentes técnicas de imagen cardiaca que contribuyen en el diagnóstico. Este es un reporte de caso de disección atrial izquierda como complicación de una cirugía de la válvula mitral en un paciente de 47 años de edad, quien fue intervenido quirúrgicamente por una endocarditis infecciosa. En este caso evaluamos el papel de dos técnicas de imagen cardiaca, la ecocardiografía transesofágica y la tomografía cardiaca.

ABSTRACT
Atrial dissection is a rare entity that generally occurs as a complication of mitral valve surgery. Transesophageal
echocardiography is the most important diagnostic tool, however, we currently have different cardiac imaging
techniques that contribute to the diagnosis. This is a case report of left atrial dissection as a complication of mitral
valve surgery in a 47-year-old patient who underwent surgery for infective endocarditis. In this case, we evaluate
the role of two cardiac imaging techniques, transesophageal echocardiography and cardiac tomography.
\end{abstract}

\section{Presentación del caso}

Se trata de un paciente varón de 47 años de edad, con un tiempo de enfermedad de aproximadamente un mes, caracterizado por disnea progresiva asociada a fiebre. En el examen físico se encontró soplo diastólico en foco aórtico, signos de congestión pulmonar y bloqueo atrioventricular (BAV) de III grado en el electrocardiograma. Se realizó una ecocardiografía transtorácica y luego una ecocardiografía transesofágica (ETE) encontrando una válvula aórtica bicúspide sin rafe, con perforación del velo posterior y prolapso del velo anterior aórtico, que generaba un jet de insuficiencia severa dirigido hacia el velo mitral anterior. A nivel de la válvula mitral se encontró el velo anterior con tejido redundante, que prolapsaba en sístole hacia la aurícula izquierda, perforada a nivel central compatible con aneurisma del velo mitral anterior. Se realizaron hemocultivos resultando positivos para Estreptocos sanguinis. El paciente fue diagnosticado de endocarditis infecciosa, iniciando tratamiento antibiótico y tratamiento quirúrgico con reemplazo valvular aórtica y mitral con prótesis mecánica. En el postoperatorio inmediato su evolución fue favorable. Al cuarto día del postoperatorio presentó disnea con requerimiento de oxígeno, que progresó rápidamente a severa congestión pulmonar. En la ecocardiografía transtorácica se encontró velocidad y gra- dientes elevados a nivel de la prótesis mitral. Inmediatamente después se le realiza una ETE encontrando dentro de la cavidad auricular izquierda una imagen lineal (signo de la doble pared), que dividía a la aurícula en dos cámaras, luz verdadera y luz falsa, imagen compatible con disección atrial izquierda (Figura 1, 2 y Videos 1, 2). Un flujo turbulento ingresaba desde el ventrículo izquierdo hacia el anillo mitral posterior, disecando la pared posterior de la aurícula izquierda. La luz falsa obliteraba parcialmente la luz verdadera, lo cual explicaba el incremento de velocidad y gradientes a nivel de la prótesis mitral (Figura 3 y Video 3). La prótesis mecánica mitral presentaba adecuada motilidad de los hemidiscos, sin evidencia de masas o trombos. Inmediatamente después se le realizó una tomografía cardiaca (TC) donde se corroboró el diagnóstico (Figura 4), encontrando además imágenes de dilataciones saculares ubicados a nivel del anillo mitral posterior compatibles con pseudoaneurismas (Figura 5), los cuales transcurrían adyacentes al segmento distal de la arteria circunfleja (Figura 6 y 7), y se comunicaban directamente con la luz falsa de la disección atrial. Las venas pulmonares y el ostium de la orejuela izquierda drenaban a nivel de la luz verdadera. El paciente reingresa a sala de operaciones donde se corrobora el diagnóstico. Se realizó reconstrucción del anillo mitral con parche pericárdico bovino, cerrando así la puerta de entrada de la disección. El paciente evolucionó favorablemente siendo dado de alta 3 semanas después. 


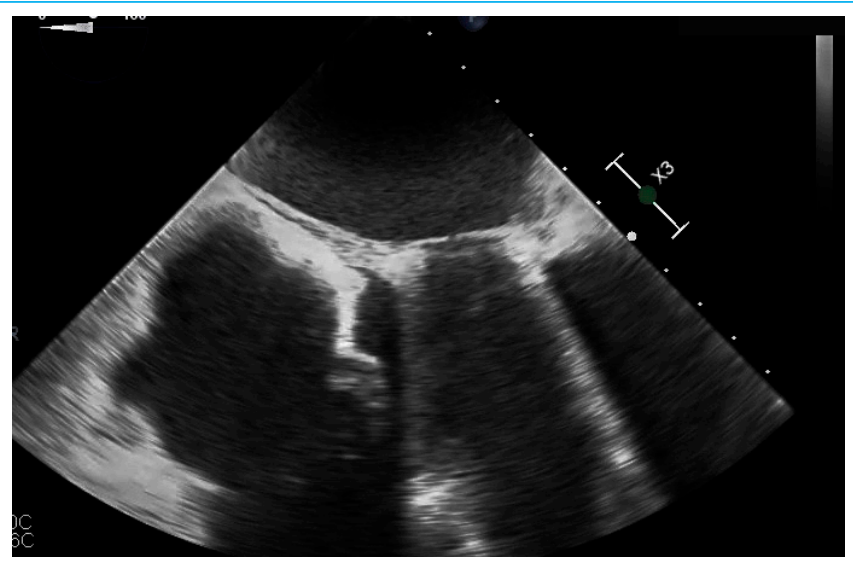

Figura 1. Ecocardiografía transesofágica en vista de 4 cámaras, donde se evidencia imagen lineal en la cavidad atrial izquierda (doble pared) correspondiente al flap de disección intimal.

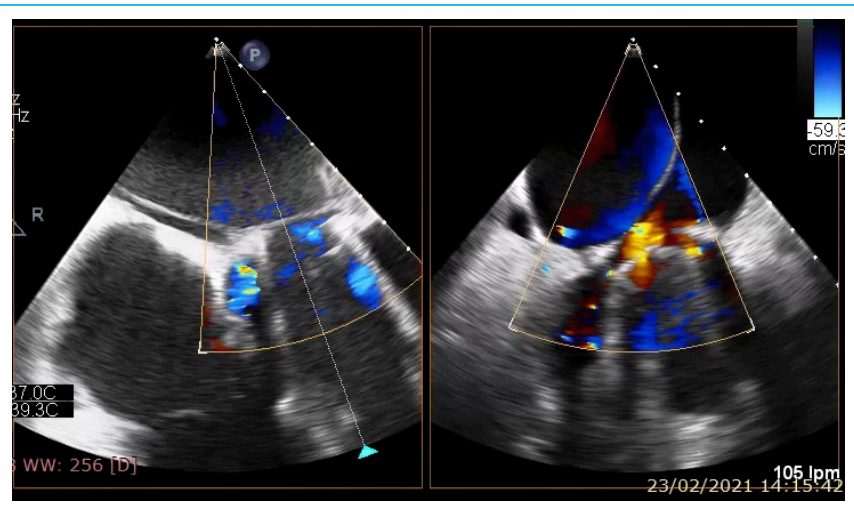

Figura 2. Ecocardiografía transesofágica en vista de 4 cámaras. Se observa flap intimal que divide en dos a la cavidad atrial izquierda, luz verdadera y luz falsa, evidenciándose en esta última, flujo turbulento en su interior.

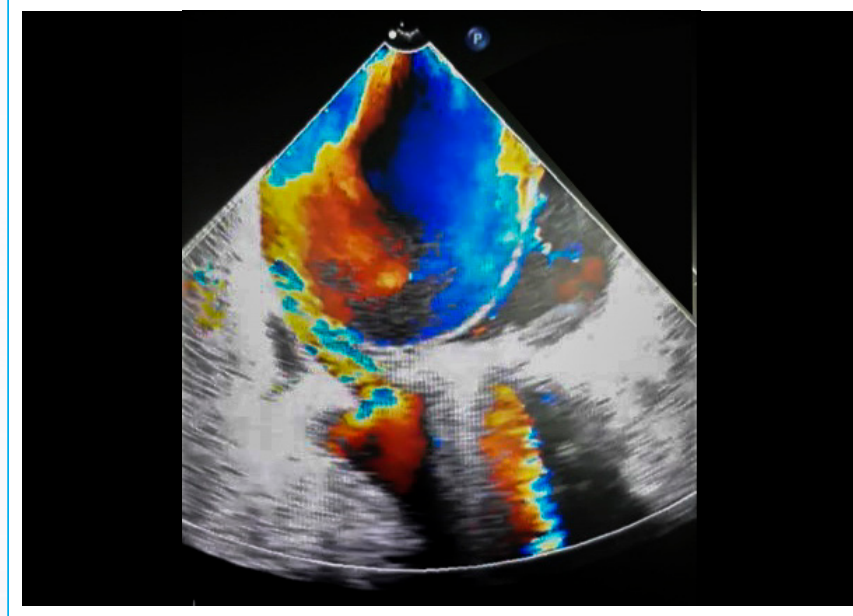

Figura 3. Ecocardiografía transesofágica, vista de dos cámaras. Se observa flujo turbulento ingresando desde el ventrículo izquierdo hacia el anillo posterior mitral, disecando la pared posterior de la aurícula izquierda. La luz falsa, obliteraba parcialmente la luz verdadera, lo cual explicaba el incremento de velocidad y gradientes a nivel de la prótesis mitral.

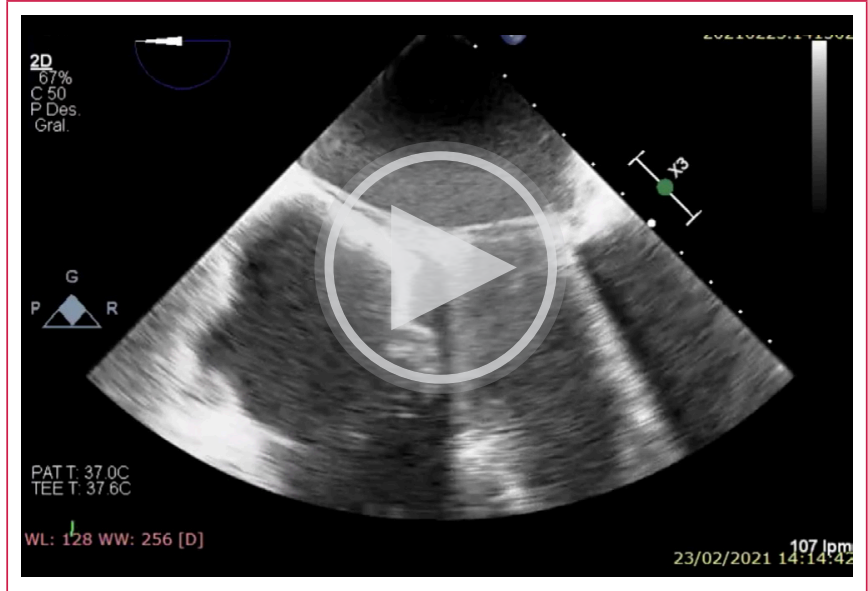

Vídeo 1. Ecocardiografía transesofágica en vista de 4 cámaras, con el flap de disección intimal. Este vídeo se corresponde con la Figura 1.

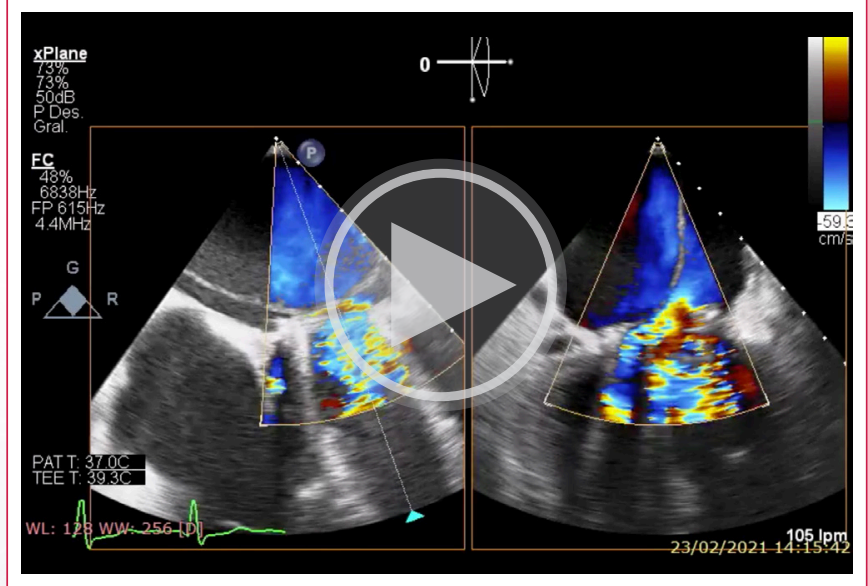

Vídeo 2. Ecocardiografía transesofágica en vista de 4 cámaras con el flap intimal de la aurícula izquierda. Este vídeo se corresponde con la Figura 2.

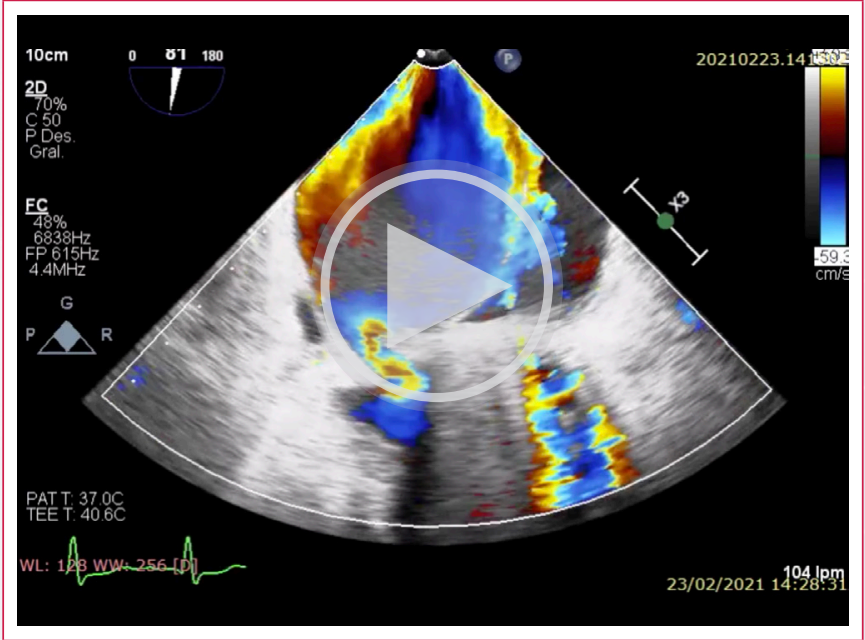

Vídeo 3. Ecocardiografía transesofágica, vista de dos cámaras. Se observa el flujo turbulento que diseca la pared posterior de la aurícula izquierda. Este vídeo se corresponde con la Figura 3. 


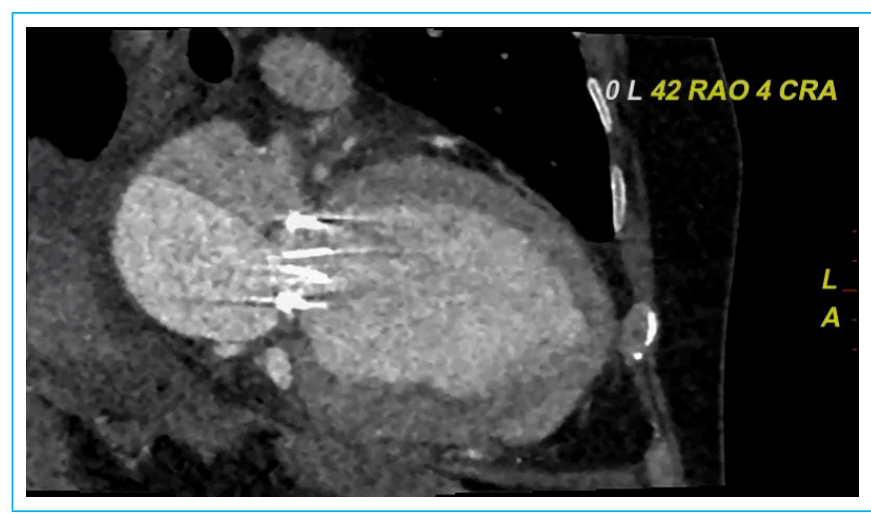

Figura 4. Tomografía cardiaca donde se observa flap de disección que divide la cavidad auricular izquierda en dos luz verdadera y luz falsa. La luz falsa ocupa aproximadamente el $70 \%$ de la cavidad.

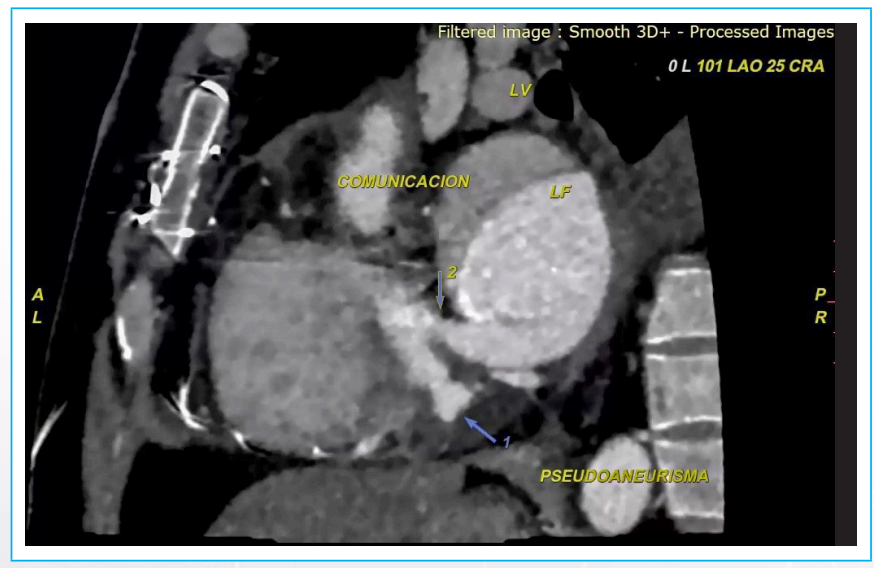

Figura 5. Tomografía cardiaca que evidencia dilataciones saculares ubicados a nivel del anillo mitral posterior compatibles con pseudoaneurismas, las cuales se comunican diorectamente con la falsa luz.

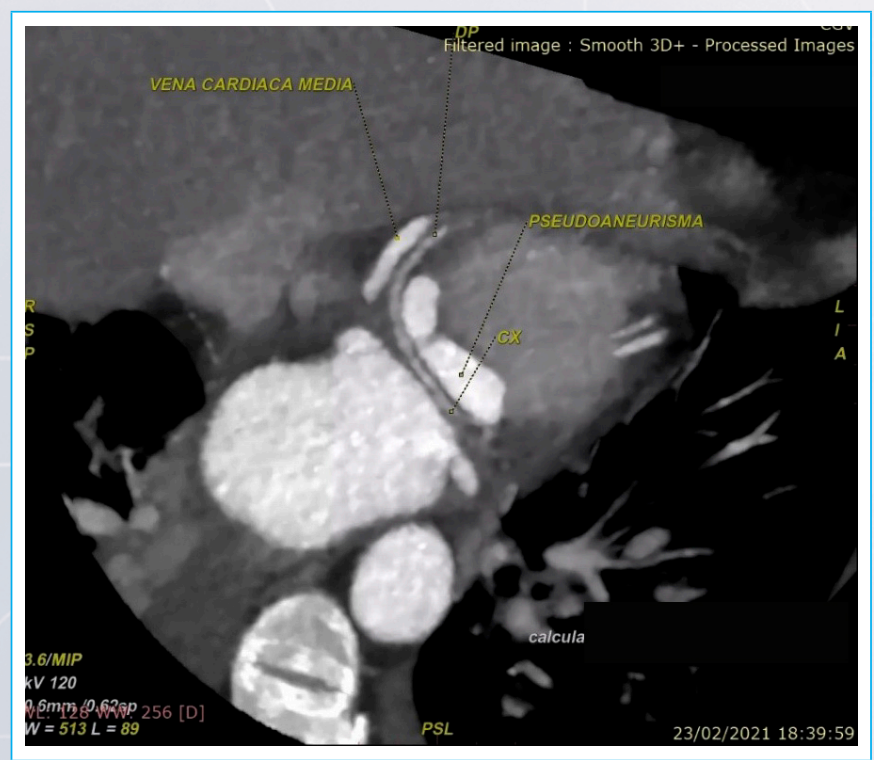

Figura 6. Los pseudoaneurismas transcurrían adyacentes al segmento distal de la arteria circunfleja.

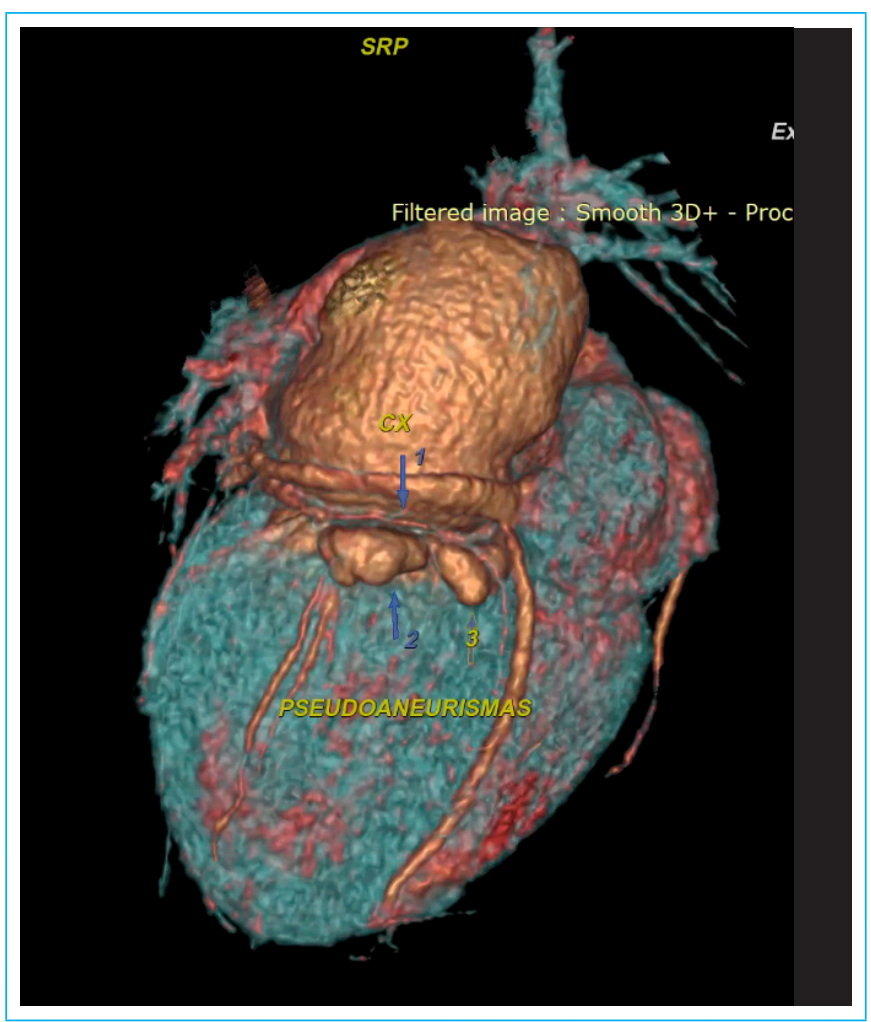

Figura 7. Reconstrucción tridimensional de tomografía cardiaca, donde se evidencia pequeñas formaciones saculares a nivel del anillo mitral posterior las cuales rodean a la parte distal de la arteria circunfleja.

\section{Discusión}

La disección atrial izquierda se define como la apertura desde el área anular mitral o tricuspídea al septum interatrial o pared atrial izquierda, creando una nueva cámara con o sin comunicación con la luz verdadera. La luz falsa aparece como un área ecolúcida que puede causar o no obliteración parcial de la luz verdadera ${ }^{(1)}$

La disección atrial izquierda se trata de una entidad rara, cuya incidencia va desde 0.16-0.84\% después de una cirugía de la válvula mitral(2,3), por lo que la mayor parte de bibliografía encontrada se trata de reporte de casos. Si bien la causa más frecuente ha sido asociada a cirugía de la válvula mitral, existen también otras causas reportadas como infarto agudo de miocardio, trauma torácico, endocarditis infecciosa, procedimientos cardiacos invasivos ${ }^{(4)}$. Todas ellas tendrían en común la lesión a nivel de la zona auriculoventricular, resultando en la separación del endocardio de la aurícula izquierda y miocardio. La disección atrial es más común a nivel de la pared posterior de la aurícula izquierda, probablemente porque esta zona está formada principalmente por músculo y escaso tejido fibroso, a diferencia del anillo anterior donde se encuentra la cortina fibrosa mitroaórtica ${ }^{(5)}$. Principalmente ocurriría debido a un debridamiento agresivo del anillo mitral posterior, incisión inadvertida, sutura inapropiada o tracción del anillo mitral posterior ${ }^{(6)}$. El cuadro clínico posee un amplio espectro, desde los cuadros asintomáticos hasta el shock cardiogénico que requiere cirugía inmediata. La ETE es el examen diagnóstico de elección ${ }^{(7)}$, el cual demuestra el flap intimal como principal criterio diagnóstico, el cual se evidencia como una imagen lineal (signo de la doble pared) que divide a la cavidad en dos luces (luz verdadera y falsa). Además, la ETE 
provee otros datos importantes como la evidencia o no de comunicación entre ambas luces, la presencia de flujo turbulento o trombo en la falsa luz, ubicación de la puerta de entrada, y obstrucción del flujo a través de la válvula mitral. Sin embargo, la ETE podría no ser diagnóstica o, como en este caso, no valorar todos los aspectos necesarios para planificar el tratamiento quirúrgico más adecuado por lo que en algunos casos se podría complementar con diferentes técnicas de imagen cardiaca como la TC o la resonancia magnética cardiaca (RMC) ${ }^{(8)}$. En este caso el uso de la TC, previo al tratamiento quirúrgico, permitió evidenciar la presencia de dos pseudonaeurismas a nivel del anillo mitral posterior que rodeaban la porción distal de la arteria circunfleja, lo que permitió una mejor planificación del tratamiento quirúrgico. Además, gracias a la mejor resolución espacial de la TC, se pudo valorar el drenaje de las venas pulmonares y de la orejuela izquierda hacia la luz verdadera. Si bien la resonancia cardiaca también se ha descrito como herramienta diagnóstica, su uso en la práctica clínica se ve limitado por su baja disponibilidad y alto costo. En nuestro caso clínico suponemos que, tanto la cirugía reciente de reemplazo de la válvula mitral y el tejido friable de la zona basal del septum ventricular, contribuyeron en la etiología de la disección atrial. La ETE nos permitió hacer el diagnóstico de forma inmediata y al pie de la cama de paciente. Sin embargo, en este caso la contribución de la TC en complementar el diagnóstico permitiendo precisar la anatomía y las complicaciones asociadas, fue de gran utilidad a la hora de planificar el tratamiento quirúrgico.

\section{Conclusiones}

La disección atrial izquierda es una entidad rara, que en la mayoría de casos se presenta como complicación de una cirugía de la válvula mitral. La ecocardiografía transesofágica constituye la principal herramienta diagnóstica, demostrando el flap intimal como criterio diagnóstico. Sin embargo, en la actualidad podemos utilizar las diferentes técnicas de imagen cardiaca, como la tomografía y la resonancia, las cuales contribuyen en la precisión diagnóstica debido a su mayor resolución espacial, por lo que recomendamos que siempre que sea posible se complemente el diagnóstico con el uso de la multimodalidad, a fin de una mejor planificación del tratamiento.

\section{Ideas para recordar}

- La disección atrial es una entidad rara que se presenta con mayor frecuencia como complicación de una cirugía de la válvula mitral.

- La ecocardiografía transesofágica constituye la herramienta diagnóstica más importante, la cual podría ser utilizada en la cabecera del paciente hemodinámicamente inestable.

- Actualmente podemos hacer uso de las diferentes técnicas de imagen, como la tomografía cardiaca y resonancia magnética cardiaca, las cuales contribuyen a brindar mayor precisión anatómica y evaluar otras posibles complicaciones asociadas, o confirmar el diagnóstico.

\section{Bibliografía}

1. Gallego P, Oliver J, González A, et al. Left Atrial Dissection: Pathogenesis, Clinical Course, and Transesophageal Echocardiographic Recognition. J Am Soc Echocardiogr 2001;14:813-20.

2. Fukuhara S, Dimitrova K, Geller C, et al. Left Atrial Dissection: Etiology and Treatment. Ann Thorac Surg 2013;95:1557- 62.

3. Martinez-Selle's M, García-Fernandez M, Moreno M, et al. Echocardiographic Features of Left Atrial Dissection. Eur J Echocardiogr 2000;1:147-50.

4. Fukuhara S, Dimitrova K, Geller C, et al. Left atrial dissection: an almost unknown entity. Interactive CardioVascular and Thoracic Surgery 2015;20:96-100.

5. Suraci N, Mihos CG, Volsky A, Santana O. Left atrial dissection: A rare entity Echocardiography 2019;36:1598-1600.

6. Arora D, Mishra M, Mehta Y, Trehan N. A Case of Left Atrial Dissection after Mitral Valve Replacement. Ann Card Anaesth 2018;21:297-9.

7. Moise OL, Loghin C, Tran SF, Estrera AL, Reece TB, Oxorn DC, Sheinbaum R. Left Atrium Dissection: A Rare Cardiac Surgery Complication. J Cardiothorac Vasc Anesth. 2017;31:1119-1122.

8. Lavorgna A, Villani C, Fabiani D, Napoletano C. A Rare Case of Left Subacute Atrial Dissection: Multimodality Imaging Approach. J Cardiovasc Echogr. 2019;29:26-28. 